\title{
Ultrasonography-guided invasive procedures of the temporomandibular joint
}

\author{
Kaan Orhan ${ }^{1,2,3}\left[\right.$ - Ingrid Rozylo-Kalinowska ${ }^{4}$
}

Received: 3 May 2019 / Accepted: 19 December 2020 / Published online: 3 February 2021

(๑) The Author(s), under exclusive licence to Springer Nature Switzerland AG part of Springer Nature 2021

\begin{abstract}
Temporomandibular joint (TMJ) complex is one of the most complex joints, which connects the mandible to the skull. This kind of joint system has the ability to perform complex and bilateral movements. Due to complex anatomy, there is a need for a thorough investigation to find the correct etiology of TMJ disorders. For a thorough investigation, starting from clinical examination to imaging strategies should be performed meticulously. Radiographic imaging is an important element in the correct diagnosis. Because of the anatomic complexity of the temporomandibular joint and its proximity to the temporal bone, mastoid air cells, and auditory structures, imaging of the joint structures should also be investigated. Therefore, careful clinical and radiological examinations are essential in the evaluation of TMJ. The manuscript examines the potential use and technique of ultrasonography (USG) in fine-needle aspiration biopsy and intra-articular sodium hyaluronate/steroid injection.
\end{abstract}

Keywords Ultrasonography · Temporomandibular joint · Temporomandibular joint disorders · Interventional radiology · Diagnostic imaging · Fine-needle biopsy · Hyaluronic acid

Kaan Orhan

cal153@yahoo.com

Ingrid Rozylo-Kalinowska

radiologia.stomatologiczna@umlub.pl

1 Department of Dentomaxillofacial Radiology, Faculty of Dentistry, Ankara University, Ankara, Turkey

2 OMFS IMPATH Research Group, Department of Imaging and Pathology, Faculty of Medicine, University of Leuven and Oral and Maxillofacial Surgery, University Hospitals Leuven, Leuven, Belgium

3 Medical Design Application and Research Center (MEDITAM), Ankara University, Ankara, Turkey

4 Department of Dentomaxillofacial Radiology, Medical University of Lublin, Poland ul. Karmelicka 7 20-081, Lublin, Poland 


\section{Quick reference/description}

Several imaging techniques are being used for temporomandibular joint (TMJ) imaging: conventional radiography, plain and panoramic radiography, computerized tomography (CT) scan, cone-beam tomography (CBCT), arthrography, magnetic resonance (MRI), axiography and also ultrasonography. For bone components imaging, conventional radiographies, CT and CBCT modalities are being used, while for soft tissue imaging, MRI is the gold standard for TMJ imaging. MRI provides a direct form of soft tissue visualization with excellent spatial and contrast resolution on sagittal and coronal MR images and essential information about position, morphology, and signal intensity characteristics of the TMJ structures. Ultrasonography, a prevalent method of diagnostic imaging, utilizes the physical properties of ultrasound for dynamic realtime examination. Considering the various advantages of ultrasonography, it can also be used for TMJ imaging in terms of soft tissue in the diagnosis of pathologies of the TMJ and for guidance during TMJ diagnostic and therapeutic procedures.

\section{Indications}

As far as TMJ is concerned, the following applications were described:

- Joint effusion

- Internal derangement and disc displacement, mainly anterior with and without reduction, no studies reported data on lateral or posterior disc displacement

- Osteoarthritis including condylar erosion

- Rheumatoid, psoriatic, and juvenile idiopathic arthritis with TMJ involvement as well as polyarthritis

- Joint function basing on condylar translation range

- Condylar movement using duplex Doppler

- TMJ dislocation

- Guidance in fine-needle aspiration cytology (FNAC)

- Guidance for TMJ arthrocentesis

- Guidance in TMJ injections, e.g., with steroids.

There are contraindication neither for ultrasound scanning nor for special patient preparation required. Using of a gel standoff pad may be necessary in case of very superficially located lesions, but a thick layer of gel with limited pressure on the probe may be applied in its stead.

\section{Materials/instruments}

Materials for the following procedures are: 
- Water-soluble gel

- Sterile gloves

- Skin disinfectants

- Local anesthetic solution with 1:100,000 epinephrine

- Sticking plaster

- Sodium hyaluronate (procedure III-ultrasonography-guided sodium hyaluronate and steroid injection)

Instruments for the following procedures are:

- Ultrasound machine

- Transducer (linear probe)

- Gel standoff pad

- 19/23/27-gauge needles

- Dermal pen

- Soft ruler

- Examination bed

\section{Procedure}

In clinical practice, ultrasonography uses acoustic waves with frequency ranging from 2 to $20 \mathrm{MHz}$. The source of the ultrasound is an ultrasonography probe. This probe is also known as a transducer that comprises a piezoelectric device, which emits acoustic waves and also receives acoustic signals returning from an examined object (echoes).

The applied ultrasonography probes account for the feasibility of an ultrasound unit. High-frequency linear probes (over $12 \mathrm{MHz}$ ) with a relatively small 'footprint' are used in the diagnostic imaging of TMJ, as they provide high-resolution images with a relatively low penetration depth that is adequate for evaluation of superficially located joints. Intraoral probes, such as an intraoperative 'hockey stick' probe, are useful in the imaging of masticatory muscles.

\section{Ultrasonography of the TMJ}

Ultrasonography of the TMJ is a useful method of diagnostic imaging of the TMJ. A $3 \mathrm{D}$ ultrasound probe can help to overcome the issue of obtaining images that are never truly sagittal or transverse while using a 2D transducer. The technique of TMJ ultrasonography is as follows:

1. Ask the patient to lay on a special examination bed or ask the patient to sit in an upright sitting position.

2. Apply generous amount of a water-soluble gel on the patient's skin within the area of interest to remove air bubbles between the skin and the probe. 
3. Place the transducer parallel to the Frankfurt horizontal plane (Fig. 1a, b) and also at an angle of $60-70^{\circ}$ to the plane (Fig. 1c, d), making it parallel to the ramus of mandible, in the open and closed mouth position. The probe should be moved gently over the area of concern.

4. During the ultrasound examination, several images are captured including range of condylar movement range.

5. Ultrasound scanning of masticatory muscles can also be performed.

\section{Fine-needle Aspiration Biopsy}

Histopathological evaluation is considered as the gold standard for diagnosing TMJ pathologies. For procuring a pathological specimen and performing subsequent histopathological assessment, fine-needle aspiration biopsy is an effective approach.
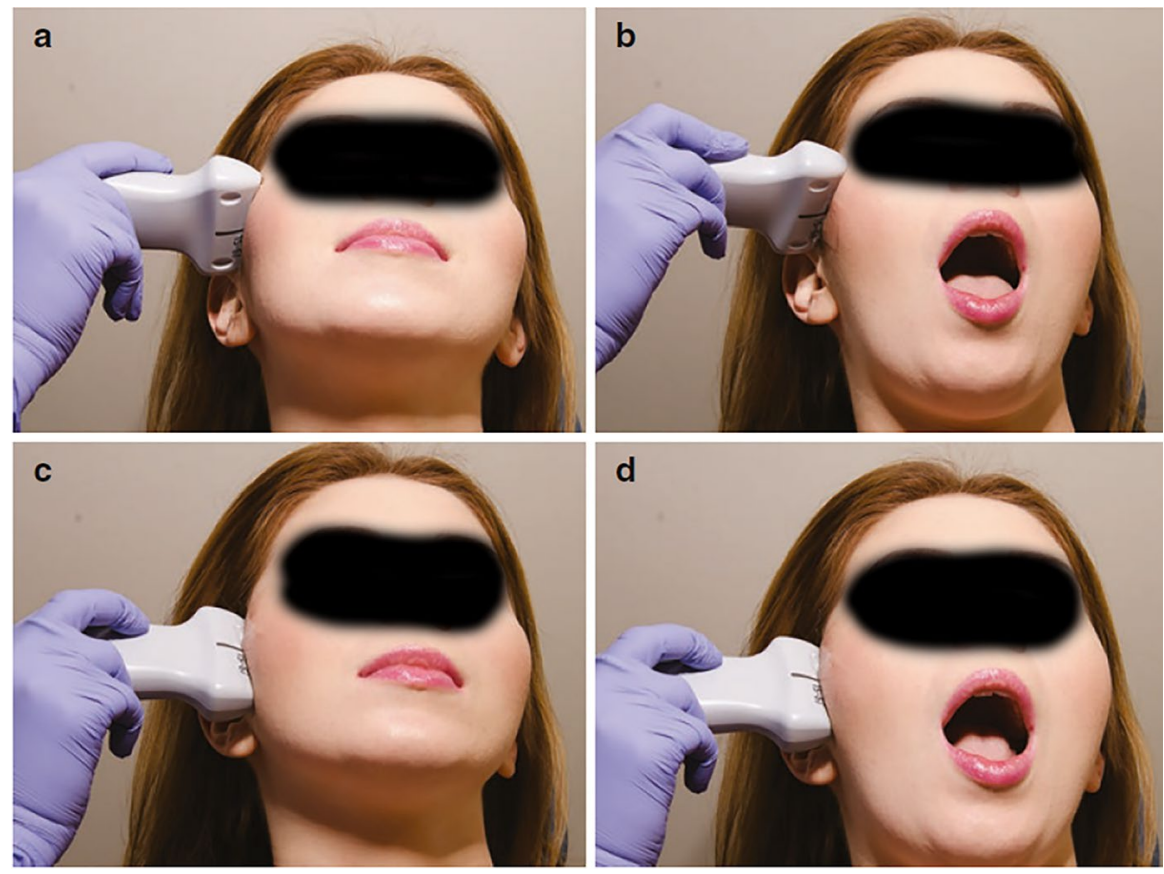

Fig. 1 Ultrasound examination of the right TMJ. The transducer is placed parallel to the Frankfurt horizontal plane in closed (a) and open (b) mouth position as well as at 60-70 degrees to the plane, parallel to the ramus of mandible, both in closed (c) and open (d) mouth position 


\section{Ultrasonography-guided fine-needle aspiration biopsy}

To ensure accurate results of fine-needle aspiration biopsy, a primary method of gaining access to the pathology is ultrasonography guidance. The advantages of ultrasonography-guided fine-needle aspiration biopsy are:

- Easy access to ultrasound technology

- Ease of use for the operator

- Minimally invasive procedure

- Good tolerance by the patients

Ultrasonography-guided fine-needle aspiration biopsy is a minimally invasive procedure and should be performed under sterile conditions. This procedure can be performed by a single operator or by two operators. In the two-operator technique, one operator conducts the ultrasonography and guides the biopsy, while the other operator performs the biopsy. The technique of ultrasonography-guided fine-needle aspiration biopsy of TMJ is as follows:

1. Perform the ultrasonography of the TMJ after topical application of the watersoluble gel for a complete examination of the TMJ anatomy and the pathology.

2. Wipe the ultrasonography gel from the area of interest. Wear sterile gloves and achieve antisepsis by cleaning the region with skin disinfectants.

3. Administer a local anesthetic solution containing 1:100,000 epinephrine carefully by injecting it slowly with a 27 -gauge needle into the TMJ capsule. The careful and slow administration of local anesthetic prevents discomfort during the procedure.

4. Palpate the TMJ region thoroughly. During palpation, instruct the patient to open and close the jaws slowly for identifying the zygomatic arch and mandibular condyle.

5. To locate the entrance point of the needle, use a soft ruler and a dermal pen. Align the ruler from the outer cantus of the eye to the tip of the outer ear tragus. On this line, mark a point $10 \mathrm{~mm}$ anterior from the tragus tip. Mark another point $2 \mathrm{~mm}$ below the first point on a second imaginary line parallel to the first line. The second point is the entrance point of the needle. The distance from the point on the skin to the TMJ is approximately $25 \mathrm{~mm}$. Therefore, use a 23- or 27-gauge needle. Instruct the patient to open the jaws wide to increase the joint space.

6. The operator then positions the ultrasonography probe anterior to this entrance point. After visualizing the TMJ structures and pathology, the needle is inserted from the specified point with the bevel toward the TMJ (Fig. 2a). After some advancement, the needle tip should be detected through ultrasonography (Fig. 2b). Then advance the needle into the structures in a posterior and superior route, to reach into the pathology. During needle advancement, coordination is necessary between the operators.

7. After reaching the pathology, penetrate the needle into the center of the lesion. Under mild vacuum applied with the plunger biopsy, the specimen is obtained with light forward-backward movements of the needle. The needle is then 


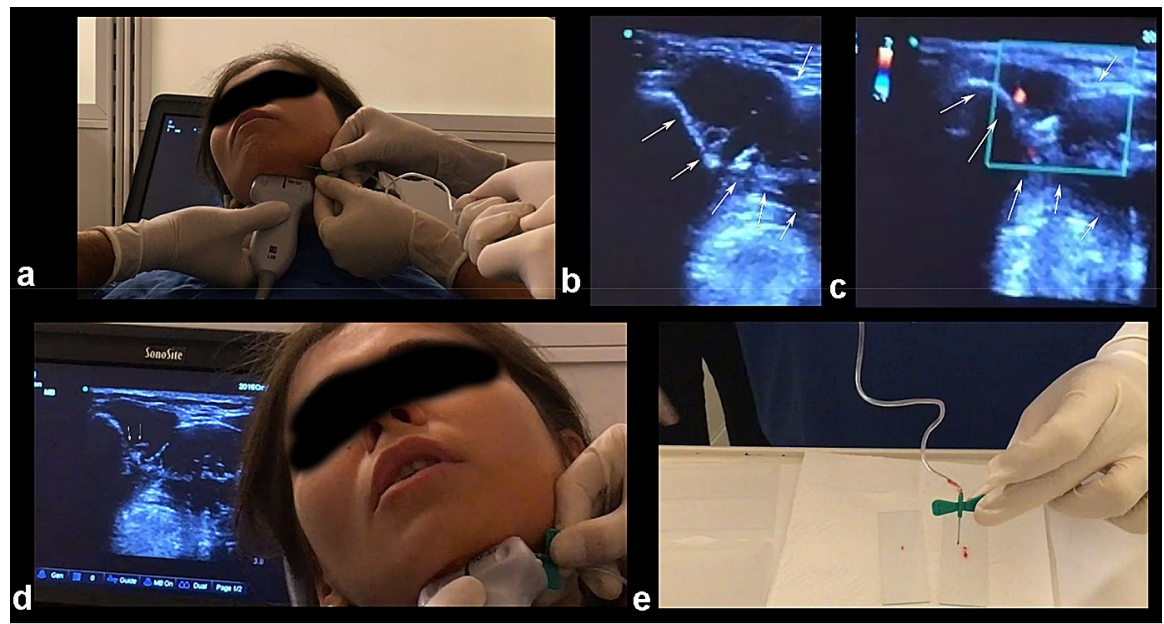

Fig. 2 Fine-needle aspiration biopsy of a lymph node mass (a) the entrance of needle with USG guidance, (b) mass in B mode showing extensive large mass with hypoechoic image, (c) mass in color Doppler mode showing minimal vascularization, (d) US image showing needle (white arrow) and the mass together with the positioning of the needle, $\mathbf{c}$ the specimen for histopathological examination

removed, while the mild vacuum continues, and the specimen is spread out on a lamella. Thereafter, the specimen is sent for histopathological analysis.

8. Close the area using a sticking plaster with light pressure after the biopsy to avoid hematoma formation. Advise intermittent cold-pack applications and antiinflammatory medication in the postoperative period.

\section{Intra-articular sodium hyaluronate and steroid injection}

Sodium hyaluronate is similar to the synovial fluid produced in the TMJ, which lubricates and absorbs trauma to the TMJ. For treatment of TMJ osteoarthritis, the use of sodium hyaluronate is prevalent. It is effective in reducing TMJ pain due to intra-articular derangements. Besides, the injection of a corticosteroid into the affected jaw joint can help to reduce inflammation and pain in the temporomandibular joint associated with chewing, talking, or yawning. This procedure can also help those suffering from chronic jaw pain. Temporomandibular joint arthritis in children with juvenile idiopathic arthritis responds well to intra-articular corticosteroid injection, especially when it is administered early stages.

The intra-articular administration of sodium hyaluronate or steroid injection into the TMJ can be performed under ultrasonography guidance for accurate results.

\section{Ultrasonography-guided sodium hyaluronate and steroid injection}

The preoperative preparation and technique of ultrasonography-guided sodium hyaluronate or steroid injection are similar to those of fine-needle aspiration biopsy. The 
injection should be performed under sterile conditions by a single operator or by two operators. In the two-operator technique, one operator conducts the ultrasonography and guides the biopsy, while the other operator administers the injection. The technique of performing ultrasonography-guided injection is as follows:

1.-6. The initial steps of ultrasonography-guided sodium hyaluronate or steroid injection are the same as steps II 1-6 of ultrasonography-guided fine needle aspiration biopsy (Fig. 3a-d). Prior to the injection, the injection site will be cleaned and numbed with a local anesthetic.
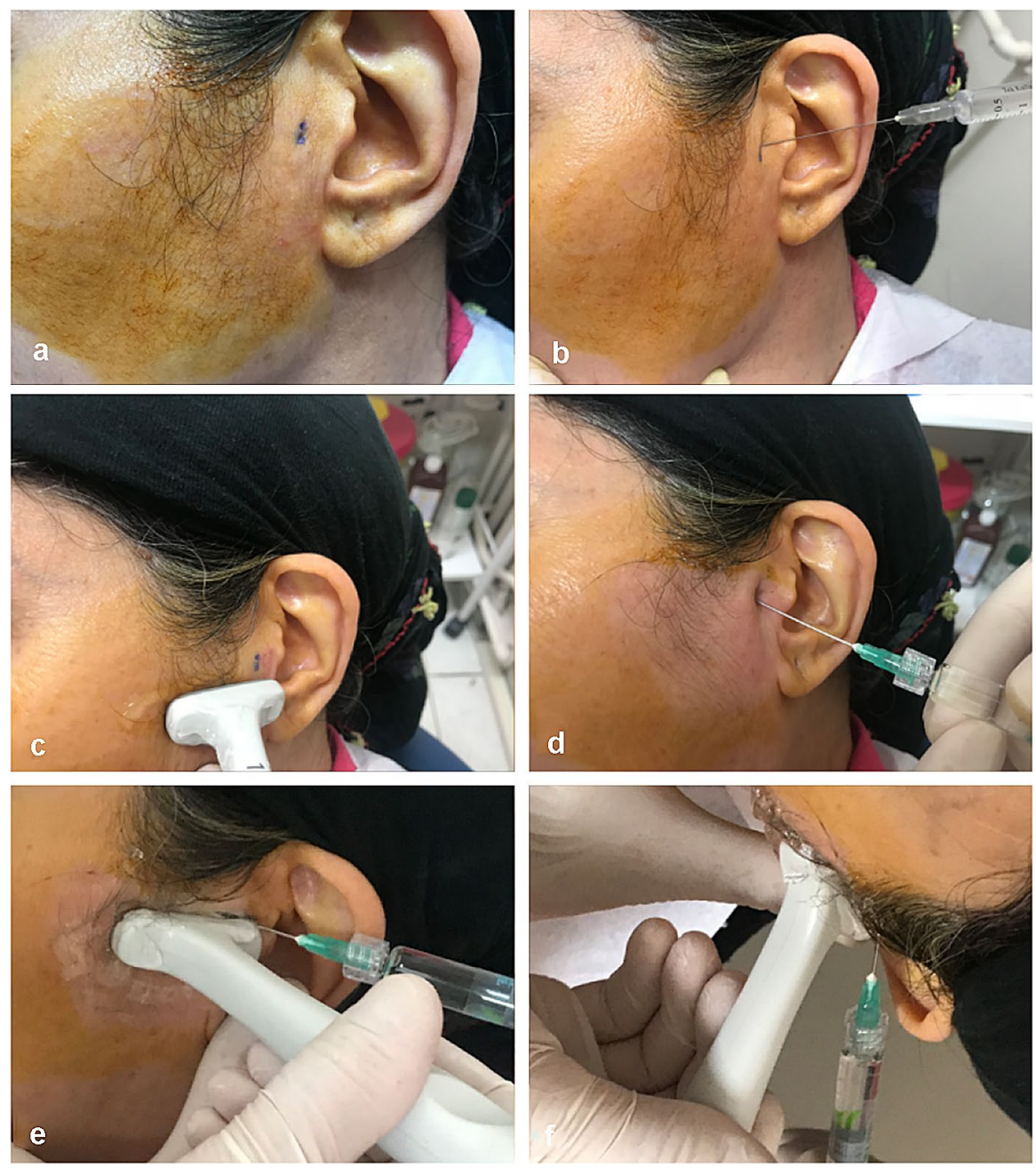

Fig. 3 Ultrasonography-guided intra-articular sodium hyaluronate injection procedure. a Identifying the entrance of the needle. b Local anesthesia application. c Ultrasonography-guided examination before entrance. d Entrance of the needle. e, f Ultrasonography-guided intervention 
7. Sodium hyaluronate preparations are usually present in ready-to-use syringes. Steroids can be also ready to use or can be prepared with syringes individually. If such syringes are used, the provided needles should be used. Alternatively, a 19-gauge needle can be used. The ultrasonography operator assists the operator administering the injection to advance the needle in the appropriate direction (Fig. 3g). Once proper placement of the needle is confirmed, the physician will slowly inject the medications. The injection will be given, while the patient is seated or prone position.

8. After reaching the upper TMJ space, $1 \mathrm{ml}$ of sodium hyaluronate or steroid is injected slowly (Fig. 3e, f, and h). Repetitive administrations can be performed for alleviation of pain.

9. Close the area using a sticking plaster with light pressure after the biopsy to avoid hematoma formation. Advise intermittent cold-pack applications and antiinflammatory medication in the postoperative period.

This procedure is a relatively safe, non-surgical treatment with minimal risks of complications. Some possible side effects include: allergic reaction-swelling, rash, or itchiness. In same patients, facial muscle weakness or numbness and dizziness can be seen.

\section{Advantages and limitations of ultrasonography}

\section{Advantages}

- High availability and easy access for imaging

- No harmful effects (even in pregnant females and children)

- High image resolution

- Real-time imaging

- Relatively cost-effective. The average cost of a CT scan in the USA is $\$ 3275$, though prices can range from $\$ 300$ to $\$ 6750$ when estimating all procedures. The average cost of an MRI in the USA is $\$ 1325$, though prices can range from $\$ 375$ to $\$ 2850$. In the meantime, the average cost of a carotid (Neck USG) Ultrasound/Doppler ranges from $\$ 217$ to $\$ 497$ in the USA.

- Portable machine

- Can repeat and easy to store

\section{Limitations}

- High dependency on operator's skills and experience

- Cannot be used in areas that contain gas (such as lungs). Cannot demonstrate structures located behind the intact bone surface due to full reflection of the beam against the dense outer bone cortex

- In osteoarthritis, the available area for penetration with an ultrasound beam is reduced due to the decrease in joint space width and bony spur formation

- Does not contain as much detail as other advanced imaging techniques. A deeper imaging requires a lower frequency which causes lower resolution. 
- More research is needed to determine whether or not TMJ pathology is detectable comparable to DC/TMD criteria.

- Anisotropy. Simply this means a structure is highly reflective to ultrasound. This occurs with nerves, tendons, and needles. The US beam must be at or close to perpendicular to the structure for the beam to 'bounce' back to the probe for an image to be created.

- Training. Ultrasound techniques require improved anatomical knowledge and a formal educational program.

\section{Pitfalls and complications}

- As ultrasound is a dynamic real-time examination, the images obtained are never truly transverse or sagittal. In diagnostics of TMJ is that it is not possible to demonstrate structures located behind the intact bone surface, as the beam is fully reflected off dense outer cortex.

- US can allow visualization of the TMJ structures but did not allow confirmation of clinical diagnosis by DC/TMD.

- Normal musculoskeletal anatomy can be misinterpreted as a manifestation of a TMJ disease. There are examples of normal musculoskeletal structures, which may mimic disease on the US, particularly to the inexperienced observer.

- Inadequate angulation of the probe to obtain diameters and cross-sectional areas, especially when evaluating TMJ disc. Diameters and cross-sectional areas are used in the US as adjuvants to objectively access thickening or thinning of TMJ disc. Care should be taken to avoid incorrect estimates. In order to correctly obtain anteroposterior diameters and cross-sectional areas, the angle of insonation must be positioned orthogonal to the long axis of the condyle.

- The echogenicity of tendons, ligaments, nerves, and muscles is variable and depends on the angle of insonation which causes a characteristic known as anisotropy. The TMJ capsule tear or disturbance can be seen as decreased echogenicity. However, hypoechogenicity caused by anisotropy is artifactual and can be differentiated from a true abnormality because it produces a more homogeneous hypoechoic appearance that can be minimized when the insonating beam is perpendicular to the muscle fibers.

- Temporary pain or swelling of the affected joint can occur after an intra-articular injection.

- In some patients, facial muscle weakness, numbness, dizziness, or allergic reactions can be seen.

- Needle fracture is a possible but rare complication in US-guided FNAB.

- Although very rare, several cases were presented with adverse skin reactions to ultrasound gel. 


\section{Cleaning/disinfection of ultrasound system for COVID-19}

Transducers must be disinfected between each patient since they can carry pathogens such as human papilloma virus (HPV). Ultrasound Working Group of the European Society of Radiology has prepared set of prevention and control recommendations, which is also applicable for COVID-19. Specific measures are recommended by American Institute of Ultrasound in Medicine (AIUM) for COVID-19. According to AIUM, all ancillary equipments of the ultrasound system should be cleaned. A physical barrier between the console and the operator can be used in addition to cleaning with low-level disinfectant. A dedicated transducer and scanner for COVID-19 suspected or positive patients should be used, if possible. A lowlevel disinfectant (quaternary ammonium) sprays or wipes should be used with soap/ water in order to clean the transducers. Intraoral transducers are internal transducers; thus, they should be disinfected with high-level disinfection following each examination. An antiseptic agent impregnated towel (such as hypochlorite/hypochlorous acid, phenol/phenolate, orthophthalaldehyde, peracetic acid or glutaraldehyde) and also a type $\mathrm{C}$ ultraviolet light can be used for that disinfection process.

\section{Further reading}

1. Ahuja A, Evans R (2013) Practical head and neck ultrasound. Cambridge University Press, Cambridge

2. Assaf AT, Kahl-Nieke B, Feddersen J, Habermann CR (2013) Is high-resolution ultrasonography suitable for the detection of temporomandibular joint involvement in children with juvenile idiopathic arthritis? Dentomaxillofac Radiol 42(3):20110379

3. Bas B, Yılmaz N, Gökce E, Akan H (2011) Ultrasound assessment of increased capsular width in temporomandibular joint internal derangements: relationship with joint pain and magnetic resonance grading of joint effusion. Oral Surg Oral Med Oral Pathol Oral Radiol Endod 112(1):112-117

4. Brandlmaier I, Rudisch A, Bodner G, Bertram S, Emshoff R (2003) Temporomandibular joint internal derangement: detection with $12.5 \mathrm{MHz}$ ultrasonography. J Oral Rehabil 30(8):796-801

5. Cakir-Ozkan N, Sarikaya B, Erkorkmaz U, Aktürk Y (2010) Ultrasonographic evaluation of disc displacement of the temporomandibular joint compared with magnetic resonance imaging. J Oral Maxillofac Surg 68(5): 1075-1080

6. Chasset F, Soria A, Moguelet P et al (2016) Contact dermatitis due to ultrasound gel: a case report and published work review. J Dermatol 43:318-320

7. Dayisoylu EH, Cifci E, Uckan S (2013) Ultrasound-guided arthrocentesis of the temporomandibular joint. Br J Oral Maxillofac Surg 51(7):667-668

8. Díaz DZR, Müller CEE, Gavião MBD

9. Emshoff R, Bertram S, Rudisch A, Gassner R (1997) The diagnostic value of ultrasonography to determine the temporomandibular joint disc position. Oral Surg Oral Med Oral Pathol Oral Radiol Endod 84:688-696

10. Emshoff R, Brandlmaier I, Bodner G, Rudisch A (2003) Condylar erosion and disc displacement: detection with high-resolution ultrasonography. J Oral Maxillofac Surg 61(8):877-881

11. Emshoff R, Jank S, Bertram S, Rudisch A, Bodner G (2002) Disk displacement of the temporomandibular joint: sonography versus MR imaging. AJR Am J Roentgenol 178(6):1557-1562

12. Emshoff R, Jank S, Rudisch A, Walch C, Bodner G (2002) Error patterns and observer variations in the high-resolution ultrasonography imaging evaluation of the disk position of the temporomandibular joint. Oral Surg Oral Med Oral Pathol Oral Radiol Endod 93(3):369-375

13. Gateno J, Miloro M, Hendler BH, Horrow M (1993) The use of ultrasound to determine the position of the mandibular condyle. J Oral Maxillofac Surg 51:1081-1086

14. Hechler BL, Phero JA, Van Mater H, Matthews NS (2018) Ultrasound versus magnetic resonance imaging of the temporomandibular joint in juvenile idiopathic arthritis: a systematic review. Int J Oral Maxillofac Surg 47(1):83-89 
15. Ilhan B, Bayrakdar İS, Orhan K (2020) Dental radiographic procedures during COVID-19 outbreak and normalization period: recommendations on infection control. Oral Radiol 36:395-399. https://doi. org/10.1007/s11282-020-00460-z

16. Jank S, Emshoff R, Norer B, Missmann M, Nicasi A, Strobl H, Gassner R, Rudisch A, Bodner G (2005) Diagnostic quality of dynamic high-resolution ultrasonography of the TMJ-a pilot study. Int J Oral Maxillofac Surg 34(2):132-137

17. Kaya K, Dulgeroğlu D, Unsal DS, Babadağ M, Tacal T, Barlak A (2010) Diagnostic value of ultrasonography in the evaluation of the temporomandibular joint anterior disk displacement. J Cranio-Maxillofac Surg 38:391-395

18. Landes CA, Goral W, Mack MG, Sader R (2006) 3-D sonography for diagnosis of osteoarthrosis and disk degeneration of the temporomandibular joint, compared with MRI. Ultrasound Med Biol 32(5):627-632

19. Landes CA, Goral WA, Sader R, Mack MG (2007) Three-dimensional versus two-dimensional sonography of the temporomandibular joint in comparison to MRI. Eur J Radiol 61(2):235-244

20. Landes CA, Sader R (2007) Sonographic evaluation of the ranges of condylar translation and of temporomandibular joint space as well as first comparison with symptomatic joints. J Craniomaxillofac Surg 35:374-381

21. Landes CA, Walendzik H, Klein C (2000) Sonography of the temporomandibular joint from 60 examinations and comparison with MRI and axiography. J Craniomaxillofac Surg 28(6):352-361

22. Larheim TA, Abrahamsson AK, Kristensen M, Arvidsson LZ (2015) Temporomandibular joint diagnostics using CBCT. Dentomaxillofac Radiol 44(1):20140235

23. Manfredini D, Guarda-Nardini L (2009) Ultrasonography of the temporomandibular joint: a literature review. Int J Oral Maxillofac Surg 38(12):1229-1236. https://doi.org/10.1016/j.ijom.2009.07.014 (Epub 2009 Aug 22)

24. Melchiorre D, Calderazzi A, Maddali BS, Cristofani R, Bazzichi L, Eligi C, Maresca M, Ciompi M (2003) A comparison of ultrasonography and magnetic resonance imaging in the evaluation of temporomandibular joint involvement in rheumatoid arthritis and psoriatic arthritis. Rheumatology 42:673-676

25. Rozylo-Kalinowska I, Orhan K (eds.), (2019) Imaging of the temporomandibular joint, https://doi. org/10.1007/978-3-319-99468-0_9

26. Stagnitti A, Marini A, Impara L, Drudi FM, Lo ML, Lillo OG (2012) Duplex Doppler ultrasound study of the temporomandibular joint. J Ultrasound 15(2):111-114

27. Tognini F, Manfredini D, Mechiorre D, Bosco M (2005) Comparison of ultrasonography and magnetic resonance imaging in the evaluation of the temporomandibular joint disc displacement. J Oral Rehabil 32:248-253

28. Tognini F, Manfredini D, Mechiorre D, Zampa V, Bosco M (2003) Ultrasonographic vs magnetic resonance imaging findings of temporomandibular joint effusion. Minerva Stomatol 52:365-372

29. (2019) Ultrasonographic study of the temporomandibular joint in individuals with and without temporomandibular disorder. J Oral Sci. https://doi.org/10.2334/josnusd.18-0278

30. Uysal S, Kansu H, Akhan O, Kansu O (2002) Comparison of ultrasonography with magnetic resonance imaging in the diagnosis of temporomandibular joint internal derangements: a preliminary investigation. Oral Surg Oral Med Oral Pathol Oral Radiol Endod 94(1):115-121

31. Weiss PF, Arabshani B, Johnson A, Bilaniuk LT, Zarnow D, Cahill AM, Feudtner C, Cron RQ (2008) High prevalence of temporomandibular joint arthritis at disease onset in children with juvenile idiopathic arthritis, as detected by magnetic resonance imaging but not by ultrasound. Arthritis Rheum 58(4):1189-1196

32. Whaites E, Drage N (2013) Essentials of dental radiography and radiology, 5th edn. Churchill Livingstone, London

33. Yılmaz D, Kamburoğlu K (2019) Comparison of the effectiveness of high resolution ultrasound with MRI in patients with temporomandibular joint disorders. Dentomaxillofac Radiol 48(5):20180349

34. Yuan S, Magarik M, Lex AM, Fleischer AC (2016) Clinical applications of sonoelastography. Expert Rev Med Devices 13(12):1107-1117

Publisher's Note Springer Nature remains neutral with regard to jurisdictional claims in published maps and institutional affiliations. 